\title{
Isolated Tubercular Liver Abscess: An Entity Rarely Thought, Diagnosed on Cytology
}

\author{
Shalini Bahadur, Aastha Narula*, Priyanka Anand and Namrata Nargotra \\ Dept. of Pathology NDMC and Hindu Rao Hospital, New Delhi, India
}

\begin{abstract}
Introduction: Tuberculosis is a rare cause of liver abscess in the Indian subcontinent even though the disease itself has high prevalance rates here. Isolated tubercular liver abscess presenting without any other foci of infection is a rare presentation with a documented prevalence of $0.34 \%$. In an adult immune competent patient diagnosis can be challenging as it is likely to be confused with pyogenic and amoebic liver abscess or hepatoma.

Case History: A 30 year old female patient presented with complaints of low grade fever, vague abdominal pain of 4 months duration, breathlessness and loss of appetite for 1 month. Past or family history was non-contributory. Ultrasonography abdomen revealed a small hypoechoic lesion in sub-hepatic region of size $49 \times 20 \mathrm{~mm}$ suggestive of a liver abscess. Computerized Tomographic scan images confirmed loculated irregular collections in and around left lobe of liver and Reidel's lobe. Fine needle aspiration cytology from the lesion revealed ill formed granulomas on a necrotic background. Zeihl Neelson staining was positive for acid fast bacilli.

Conclusion: Isolated tubercular liver abscess is a rare entity, particularly in immunocompetent individuals. Symptomatology and radiologic findings may not be contributory. Diagnosis of this entity rests on the demonstration of acid fast bacilli in material obtained. FNAC is a simple minimally invasive, cost effective procedure which helps attain the same.
\end{abstract}

\section{Keywords: Liver Abscess, Tuberculosis, Rare Entity, Cytology}

\section{Abbreviations}

FNAC: fine needle aspiration cytology

USG: ultrasonography

CT: computerized tomography

MGG: May Grunwald Geimsa

ZN: Ziehl Neelson

AFB: acid fast bacilli

HIV: human immunodeficiency virus

\section{Introduction}

Liver is a frequently involved intra-abdominal organ susceptible to the development of abscess for a variety of causes chiefly organisms, be it parasitic (amoebic), fungal or bacterial (including mycobacterial). ${ }^{[1]}$ In the Indian subcontinent Tuberculosis continues to be a major public health problem and is known to exist in two forms pulmonary and extra-pulmonary. Hepatic involvement in tuberculosis has been associated with both. Bacilli from respiratory and gastro-intestinal lesions reach via Hepatic vein or Portal vein. Isolated tubercular liver abscess is a rare entity seen in $0.34 \%$ of cases with hepatic tuberculosis. ${ }^{[2]}$

Tuberculosis is a rare cause of liver abscess in the Indian subcontinent even though the disease itself has high prevalance rates here. Isolated tubercular liver abscess presenting without any other foci of infection is a rare presentation with a documented prevalence of $0.34 \%$. In an adult immune competent patient diagnosis can be challenging as it is likely to be confused with pyogenic and amoebic liver abscess or hepatoma.

\section{Case Report}

A 30 year old female patient presented to the medicine outpatient department with complaints of low grade fever and vague abdominal pain of 4 months duration. Occasional episodes of vomiting were reported during this period. She had additional complaints of breathlessness and loss of appetite for 1 month. Cough, expectoration and hemoptysis was not present. There was no past history for any chronic illness such as asthma, tuberculosis or diabetes. Prior contact with patients of tuberculosis was not documented. There was negative history of intake of anti-tubercular drugs. Her last child birth was six months prior. No history of blood transfusion or high risk behaviour was found.

General examination was non-contributory. Systemic examination showed slight dullness in right lower lung and costo-phrenic angle. Per abdomen mild tenderness in right upper quadrant was present. Laboratory investigations revealed haemoglobin of $10.7 \mathrm{gm} / \mathrm{dl}$, haematocrit $35.3 \%$, total leucocyte count of $13,000 / \mathrm{mm}^{3}$ with neutrophilia and raised liver enzymes $(\mathrm{SGOT}=153.0 \mathrm{IU} / \mathrm{L}, \mathrm{SGPT}=95.0$ 
IU/L, serum Alkaline Phosphate=150.0 IU/L). Patient was seronegative for HIV1 \& HIV2. Chest radiograph showed mild right sided pleural effusion.

Ultrasonography (USG) abdomen revealed a small hypoechoic lesion in sub-hepatic region of size 49x20mm suggestive of a liver abscess. Computerized Tomographic (CT) scan images confirmed loculated irregular collections in and around left lobe of liver and Reidel's lobe with small perigastric extention as well as thickening of omentum (Figure 4). Follow up chest radiography showed resolution of right sided pleural effusion by the time patient was investigated thoroughly. Possibility of liver abscess of infectious etiology was most probable diagnosis. Patient underwent an ultrasound guided fine needle aspiration of the hepatic lesion. Smears were stained with May Grunwald Geimsa (MGG) and showed ill formed collection of epithelioid cells, transformed macrophages and lymphocytes (Figure 1) with giant cells (Figure 2) seen on a necrotic background. Ziehl Neelson (ZN) confirmed presence of acid fast bacilli (AFB) (Figure 3) and a final diagnosis of tubercular hepatic abscess was rendered.

\section{Discussion}

Hepatic tuberculosis is not a rare disease, however isolated tubercular liver abscess is, even in a country like India

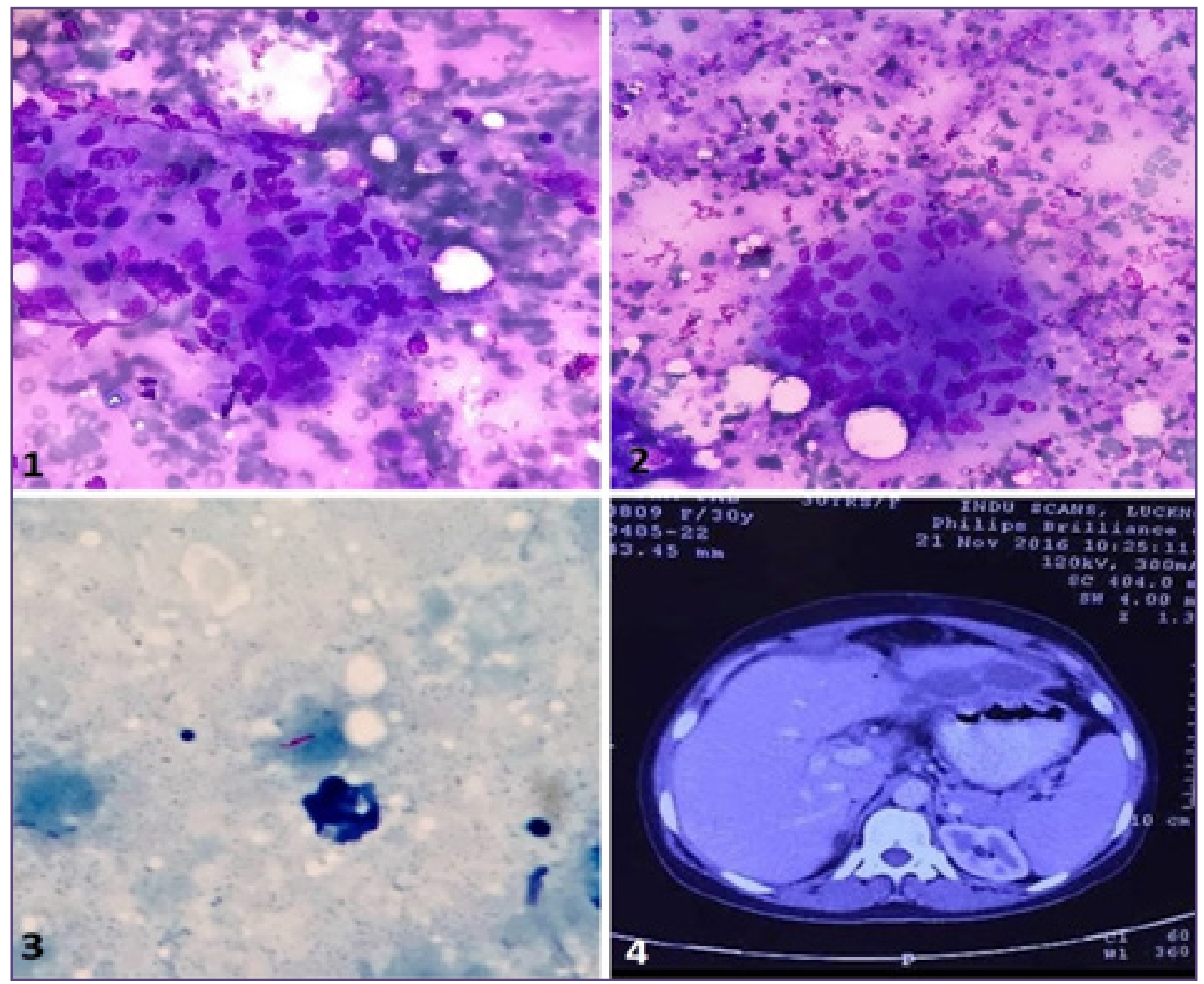

Fig. 1: ill formed granulomas consisting of epithelioid cells, lymphocytes and transformed macrophages (MGG,400X)2:photomicrograph shows langhans type of giant cell on a necrotic background(400X); 3:- acid fast bacilli seen on ZeihlNeelson stain(oil immersion 1000X); 4:- CECT axial section shows well defined hypodense loculated collection with enhancement in subcapsular region along postero-inferior margin of left lobe of liver suggestive of abscess. 
where tuberculosis is an alarming health problem. ${ }^{[3,4]}$ Low oxygen levels make liver an inhospitable organ for bacterial growth. ${ }^{[5]}$ Approximately a 100 reported cases are known in literature, to the best of our knowledge. ${ }^{[6]}$ It was first described in 1858 by Breastowe. ${ }^{[7]}$ Three forms of hepatic involvement described in tuberculosis are:- i) diffuse hepatic along with pulmonary or miliary tuberculosis ii) diffuse hepatic without pulmonary involvement iii) focal tuberculoma or abscess, the rarest of all. ${ }^{[2]}$

Involvement of liver is usually secondary to a primary focus in lungs and gastro-intestinal tract. Hematogenous dissemination of mycobacteria to liver takes place via hepatic artery or portal vein. Various authors have reported abnormalities in chest x-ray of patients with tubercular liver abscess ranging from $65 \%$ to $78 \%{ }^{[8,9,10]}$ Moreover presence of immunosuppression as a result of concomitant diabetes mellitus, AIDS, steroid therapy or chronic renal failure may further mask presenting features and delay diagnosi s.

Symptoms are usually non-specific and vague including fever, abdominal pain/discomfort and anorexia with weight loss. Jaundice is usually rare though hepatomegaly is commonly documented. ${ }^{[11]}$ Liver function test shows elevation of transaminases to variable levels with prolonged prothrombin time and reversed A:G ratio. Our case had delivered a healthy child six months prior following an uneventful pregnancy. She was immunocompetent and had presented with low grade fever and abdominal discomfort. Some loss of appetite and breathlessness possibly due to reactionary pleural- effusion had been present.

Common differential diagnosis to be considered and excluded are pyogenic and amoebic liver abscess and hepatoma. Fine needle aspiration cytology (FNAC) is an inexpensive and minimally invasive procedure that when used under USG guidance helps localize lesions and improves yield of diagnostic material. ZN stain confirms the presence of AFB on necrotic material aspirated following screening of routinely stained smears by MGG. Identification of $\mathrm{AFB}$ by $\mathrm{ZN}$ satin ranges from $0 \%-45 \%{ }^{\left[{ }^{[2]}\right.}$

Use of alternative diagnostic tests such as culture on LJ media to directly demonstrate Mycobacterium tuberculosis is practised however reported sensitivity of culture for tubercular liver abscess is $10 \% \cdot{ }^{[12]} \mathrm{PCR}$ is another diagnostic tool that enables rapid identification of the infectious organism but is costly. Due to the limitation of resources, following radiographic imaging demonstration of AFB by ZN stain aided with similar results in simultaneously put positive control smears helped us reach definitive diagnosis of tubercular liver abscess, following which appropriate medical therapy was instituted.

\section{Conclusion}

Isolated tubercular liver abscess is a rare entity, particularly in immune-competent individuals. In a country like India which has nearly $25 \%$ of global incident tuberculosis cases, its possibility should be kept in mind. Global travel trends have necessitated that elsewhere in the world too tubercular liver abscess though rare should be kept as a differential along with amoebic abscess in unknown hepatic mass lesions. Symptomatology and radiologic findings may not be contributory. Diagnosis of this entity rests on the demonstration of acid fast bacilli in material obtained and FNAC is a simple minimally invasive, cost effective procedure which helps achieve this, particularly where resources are limited and modalities like polymerase chain reaction (PCR), though available are expensive.

Isolated tubercular liver abscess is a rare entity, particularly in immunocompetent individuals. Symptomatology and radiologic findings may not be contributory. Diagnosis of this entity rests on the demonstration of acid fast bacilli in material obtained. FNAC is a simple minimally invasive, cost effective procedure which helps attain the same.

\section{Reference}

1. Kandel, R., Bhattarai, B., Maharjan, S. Unusual presentation of tuberculosis in an elderly male: a case report. Clinical Case Reports, 2015; 3(1), 43-5.

2. Bhattacharya PK, Roy A, Jamil M, Talukdar KK, Khonglah Y, Sarma K. Isolated Tubercular Subcapsular Liver Abscess: A Rare Manifestation of Extrapulmonary Tuberculosis. Journal of hepatitis. 2015;1(1):1-6.

3. Bangaroo AK, Malhotra AS. Isolated hepatic tuberculosis. J Indian Assoc Paediatr Surg. 2005;10(2):105-7.

4. Rab SM, Beg MZ. Tuberculous liver abscess. Brit J Clin Pract. 1977;31:157-8.

5. Hassani KI, Ousadden A, Ankouz A, Mazaz K, Taleb KA. Isolated liver tuberculosis abscess in a patient without immunodeficiency: A case report. World J Hepatol. 2010;2(9):354-57.

6. Yadav P, Punera DC, Nautiyal R, Kumar A. Tuberculous Liver Abscess with Right Sided Pleural Effusion. Indian Journal of Case Reports. 2015 May 30;1(2):35-7.

7. Bristowe JS. On the connection between abscess of the liver and gastrointestinal ulceration. Trans Pathol Coc. $1858 ; 9: 241-52$

8. Alvarez SZ. Hepatobiliary tuberculosis. J Gastroenterol Hepatol. 1998;13(8):833-39. 
9. Essop AR, Segal I, Posen J, Noormohamed N. Tuberculosis abscess of the liver. A case report. S Afr Med J. 1983;63(21):825-26.

10. Maharaj B, Leary WP, Pudifin DJ. A prospective study of hepatic tuberculosis in 41 black patients. Q J Med. 1987;63(242):517-22.
11. Hayashi M, Yamawaki I, Okajima K, Tomimatsu M, Ohkawa S. Tuberculous liver abscess not associated with lung involvement. Intern Med. 2004;43(6):521-3

12. Kanagaraj A, Marthandam LR, Sriramakrishnan V, Rajesh A, Meenakumari P. Tuberculous Liver Abscess. JAPI. 2008;56:645

*Corresponding author:

Dr. Aastha Narula, Dept. of Pathology, NDMC and Hindu Rao hospital, Malka ganj, New Delhi, India-110007

Phone: +91 9990033991

Email: aasthanarula87@gmail.com

Date of Submission : 04.04.2017

Date of Acceptance : 10.07.2017

Financial or other Competing Interests: None.

Date of Publication : 20.10.2017 